\title{
Cost-effectiveness analysis of a fixed-dose combination of indacaterol and glycopyrronium as maintenance treatment for COPD
}

This article was published in the following Dove Press journal: International Journal of COPD

\author{
Ming-Cheng Chan ${ }^{1, *}$ \\ Elise Chia-Hui Tan² \\ Ming-Chin Yang ${ }^{3, *}$ \\ 'Section of Chest Medicine, \\ Department of Internal Medicine, \\ Taichung Veterans General Hospital, \\ Taichung, Taiwan, Republic of China; \\ ${ }^{2}$ National Research Institute of \\ Chinese Medicine, Ministry of \\ Health and Welfare, Taipei, Taiwan, \\ Republic of China; ${ }^{3}$ Institute of Health \\ Policy and Management, National \\ Taiwan University, Taipei, Taiwan, \\ Republic of China \\ *These authors contributed equally \\ to this work
}

Correspondence: Ming-Chin Yang Institute of Health Policy and Management, College of Public Health, National Taiwan University, Room 637, No 17, Xu-Zhou Road, Taipei 10055,

Taiwan, Republic of China

Tel +88623366 8067

Fax +886223434200

Email mcyang637@ntu.edu.tw

Elise Chia-Hui Tan

National Research Institute of

Chinese Medicine, Ministry of Health

and Welfare, Room 528, No I55-I,

Section 2, Linong Street, Taipei I I22I,

Taiwan, Republic of China

Tel +88622820 I999

Fax +886228250743

Email elisetam.g@gmail.com
Objective: The aim of this study was to evaluate the cost-effectiveness of the long-acting beta-2 agonist (LABA)/long-acting muscarinic antagonist (LAMA) dual bronchodilator indacaterol/ glycopyrronium (IND/GLY) as a maintenance treatment for COPD patients from the perspective of health care payer in Taiwan.

Patients and methods: We adopted a patient-level simulation model, which included a cohort of COPD patients aged $\geq 40$ years. The intervention used in the study was the treatment using IND/GLY, and comparators were tiotropium or salmeterol/fluticasone combination (SFC). Data related to the efficacy of drugs, incidence of exacerbation, and utility were obtained from clinical studies. Direct costs were estimated from claims data based on the severity of COPD. The cycle length was 6 months (to match forced expiratory volume in 1 second $\left[\mathrm{FEV}_{1}\right]$ data), and the time horizons included 1, 3, 5, 10 years, and lifetime. Deterministic and probabilistic sensitivity analyses were conducted to test the robustness of the model results. Costs were expressed in US dollars with a discount rate of 3.0\%.

Results: Compared to tiotropium and SFC, the incremental cost-effectiveness ratios (ICERs) per quality-adjusted life year (QALY) gained of patients treated with IND/GLY were US\$5,987 and US\$14,990, respectively. One-way sensitivity analysis revealed that the improvement in $\mathrm{FEV}_{1}$ provided by IND/GLY, the distribution of patients with regard to the severity of COPD, and acute exacerbation rate ratio were the key drivers behind cost-effectiveness. Adopting a willingness to pay of US\$60,000 per QALY gained as the threshold, there was a $98.7 \%$ probability that IND/GLY was cost-effective compared to tiotropium. Similarly, there was a $99.9 \%$ probability that IND/GLY was cost-effective compared to SFC.

Conclusion: As a maintenance treatment for COPD, we consider the dual bronchodilator IND/GLY as a cost-effective strategy when compared to either tiotropium or SFC.

Keywords: COPD, LABA/LAMA dual bronchodilator, indacaterol/glycopyrronium, maintenance therapy, cost-effectiveness, ICS/LABA combination

\section{Introduction}

COPD is a prominent cause of disability and death worldwide. ${ }^{1}$ The prevalence of COPD is increasing, and the current estimates indicate that it will be the third leading cause of death by $2020 .^{2}$ COPD patients may experience acute exacerbations, which can undermine overall health status, increase the risk of mortality, and drive up medical costs. $^{3-6}$ The administration of a bronchodilator is the core treatment in COPD. Combining an inhaled corticosteroid (ICS) with a long-acting beta-2 agonist (LABA), such as the salmeterol/fluticasone combination (SFC), is recommended for patients in groups $\mathrm{C}$ (severe) and $\mathrm{D}$ (very severe) with a previous history of exacerbation. . $^{7} 8$ 
However, the inappropriate use of ICS can increase health care costs $^{9}$ as well as the risk of adverse effects, such as pneumonia, influenza, and osteoporosis. ${ }^{8,10}$

The dual bronchodilator indacaterol/glycopyrronium (IND/ GLY) is the first fixed-dose combination of a LABA and a long-acting muscarinic antagonist (LAMA) available for the maintenance treatment of COPD. Both mono-components have been approved as a once-daily maintenance treatment for COPD patients. ${ }^{11-13}$ Current guidelines recommend the combined use of inhaled LABAs and LAMAs for patients who present inadequate improvement following the administration of a single agent and have a history of infrequent exacerbations. ${ }^{8}$ In addition to IND/GLY, three more fixed-dose combinations including aclidinium/formoterol, tiotropium/olodaterol, and umeclidinium/vilanterol entered the market later.

Even though a treatment shows positive clinical benefits, it is still important to assess the potential gains in terms of improved health for the patients at an acceptable cost to the payer of the health care system. Although there are study indicating that IND/GLY is more cost-effective than SFC, ${ }^{14}$ the study design could be further improved by extracting efficacy data from just one clinical trial ${ }^{15}$ to multiple trials so that possible range of values could be included in the estimation. The efficacy of IND/GLY has been widely demonstrated in clinical trials; however, there is a dearth of information pertaining to the cost-effectiveness (CE) of IND/GLY when compared to first-line monotherapy based on LAMAs (eg, tiotropium). The objective of this study was to evaluate the CE of IND/GLY as a maintenance treatment for COPD patients under a National Health Insurance (NHI) system.

\section{Patients and methods}

\section{Study population and model overview}

Patient-level simulations were developed with 6-month cycle length using Microsoft Excel. The model is based on a simulated cohort of COPD patients aged 40 years and older, which was followed over various time horizons. A schematic illustration of the model is shown in Figure 1. The model was modified from a previous study validated in $2010^{16}$ with local empirical data, including medical cost, mortality, and epidemiology of COPD. The predictability of the model was tested by populating it with data obtained from studies on the natural

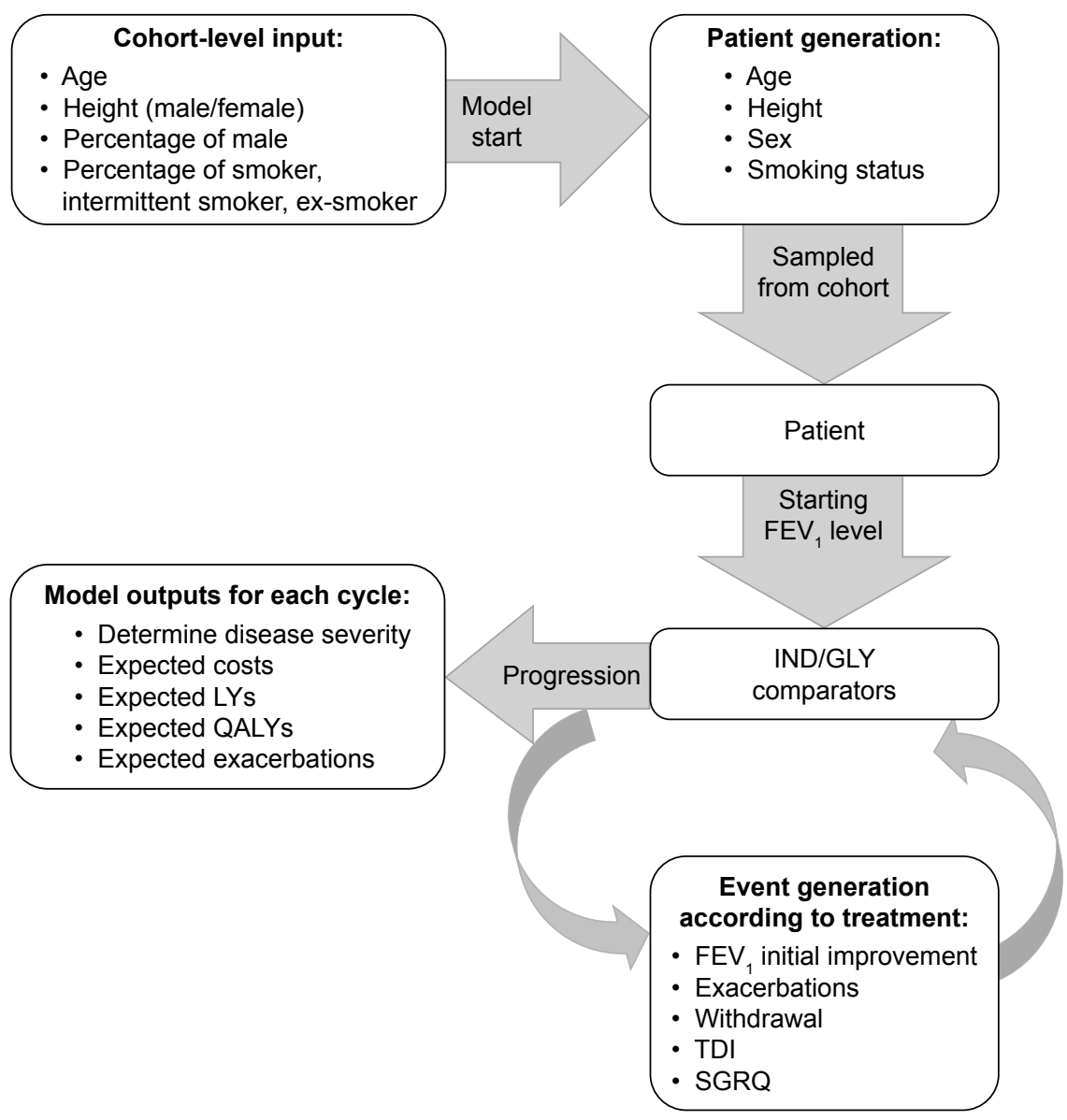

Figure I Conceptual framework of model: FEV ; IND/GLY.

Abbreviations: FEV, forced expiratory volume in I second; GLY, glycopyrronium; IND, indacaterol; LY, life year; QALY, quality-adjusted life year; TDI, Transition Dyspnea Index; SGRQ, St. George's Respiratory Questionnaire. 
history of disease as well as data obtained from clinical trials. Our results indicate that the proposed model has a generally good predictive ability, yielding in this way a high degree of external validity. Validation was conducted against two external studies. ${ }^{17,18}$ Summary statistics from these studies were extracted and input into the model. Outputs from the study included mortality rates, the rate of annual forced expiratory volume in 1 second $\left(\mathrm{FEV}_{1}\right)$ decline, and exacerbation rates.

The model outputs included costs and quality-adjusted life years (QALYs), which were calculated for individual patients and aggregated to provide the incremental $\mathrm{CE}$ ratio (ICER). The flow of individual patient was based on disease severity which was determined at each cycle based on patient's lung function. The generated patients were moved through the model one at a time according to the probabilities of developing complications, being symptomatic, or progressing, while taking into account their current treatment status. In the event of discontinuing treatment, the $\mathrm{FEV}_{1}$ level of patients would fall back to what it would have been had they never been treated. Exacerbation rates were defined on an annual basis, which in turn were adjusted to the specified cycle length. In this study, we adopted cycles of 6 months with the time horizons of 1, 3, 5, 10 years, and lifetime. This study was conducted from the perspective of Taiwan's National Health Insurance Administration (NHIA), and the discount rate of $3.0 \%$ was applied to costs and outcomes, according to the Health Technology Assessment Guidelines adopted in Taiwan. Costs and outcomes were applied at the time of the corresponding event. Costs and utility were applied simultaneously, using an additive-multiplicative method and a regression model, respectively.

The simulated index population included 1,000 cohorts, resulting in a total of $10,000,000$ patients from which to derive the cost per QALY gained ratio. This was then plotted on a CE scatterplot and CE acceptability curves (CEACs). ${ }^{19}$ The probability of death was calculated using a COPDspecific mortality hazard ratio, whereas the age of all-cause mortality was estimated for each individual using Taiwan's national life table. The parameters used in the CE model included data related to clinical efficacy, safety, utility, and exacerbation, obtained from the four clinical trials: BEACON, SHINE, SPARK, and ILLUMINATE. ${ }^{11-13,20}$ Data pertaining to the use of medical resources were obtained from National Health Insurance claims data released by the National Health Research Institute (NHRI) of Taiwan.

\section{Ethics statement}

This study was approved by the ethics board of National Taiwan University Hospital (approval number: 20141066RINB).
All participants' identifying information was scrambled in the NHI claims data.

\section{Generating patients and epidemiology data}

At the start of each patient simulation, the patient characteristics such as gender, age, height, smoking status, $\mathrm{FEV}_{1}$ status, body mass index (BMI), and baseline EQ-5D, European Quality of Life-5 dimensions (EQ-5D) levels were set (Table 1). BMI was set as $25.9 \mathrm{~kg} / \mathrm{m}^{2}$ based on the SHINE trial data. ${ }^{11}$ The baseline EQ-5D was set based on the generating characteristics of patients, and the details

Table I Clinical data and utility input

\begin{tabular}{|c|c|c|c|}
\hline Variables & Coefficient & SE & Distribution \\
\hline \multicolumn{4}{|l|}{ Covariance matrix to estimate: ${ }^{11-13,20}$} \\
\hline \multicolumn{4}{|l|}{ Height } \\
\hline Age & -0.12 & 0.01 & Normal \\
\hline Gender & -10.53 & 0.24 & Normal \\
\hline Constant & 179.47 & 0.81 & Normal \\
\hline \multicolumn{4}{|l|}{ Smoking status } \\
\hline Age & -0.02 & 0.00 & Normal \\
\hline Height & 0.00 & 0.00 & Normal \\
\hline Gender & 0.08 & 0.02 & Normal \\
\hline Constant & 1.30 & 0.17 & Normal \\
\hline \multicolumn{4}{|l|}{$\mathrm{FEV}_{1}(\%)$} \\
\hline Age & 0.08 & 0.02 & Normal \\
\hline Height & 0.01 & 0.03 & Normal \\
\hline Smoking status & 0.21 & 0.43 & Normal \\
\hline Gender & 3.90 & 0.54 & Normal \\
\hline Constant & 45.78 & 5.13 & Normal \\
\hline \multicolumn{4}{|l|}{ Annual FEV decline ${ }^{26}$} \\
\hline Smokers & 0.06 & 0.06 & Normal \\
\hline Intermittent smokers & 0.04 & 0.06 & Normal \\
\hline Ex-smokers & 0.03 & 0.05 & Normal \\
\hline \multicolumn{4}{|l|}{ FEV improvement ${ }^{11-13,30}$} \\
\hline IND/GLY & 0.19 & 0.02 & Normal \\
\hline SFC & 0.16 & 0.02 & Normal \\
\hline Tiotropium & 0.13 & 0.01 & Normal \\
\hline \multicolumn{4}{|c|}{ Utility input in EQ-5D regression ${ }^{31}$} \\
\hline Constant & 0.69 & 0.04 & Normal \\
\hline Gender (male versus female) & 0.06 & 0.01 & Normal \\
\hline Post-bronchodilator therapy & 0.00 & 0.00 & Normal \\
\hline \multicolumn{4}{|l|}{$\mathrm{FEV}, \%$ predicted } \\
\hline $\begin{array}{l}\text { Number of emergency } \\
\text { department visits not resulting } \\
\text { in hospital admission in the } \\
\text { previous year }\end{array}$ & -0.03 & 0.01 & Normal \\
\hline $\begin{array}{l}\text { Number of hospital admissions } \\
\text { in the previous year }\end{array}$ & -0.02 & 0.01 & Normal \\
\hline $\begin{array}{l}\text { Number of concomitant } \\
\text { diseases in the previous year }\end{array}$ & \multicolumn{2}{|c|}{ diseases in the previous year } & Normal \\
\hline BMI & 0.00 & 0.00 & Normal \\
\hline \multicolumn{4}{|c|}{ Excess mortality for COPD population ${ }^{27}$} \\
\hline Hazard ratio & 1.02 & 0.01 & Lognormal \\
\hline
\end{tabular}

Abbreviations: BMI, body mass index; EQ-5D, European Quality of Life-5 dimensions; FEV , forced expiratory volume in I second; GLY, glycopyrronium; IND, indacaterol; SFC, salmeterol/fluticasone combination. 
are described in the "Utility data" section. The first five of these were correlated through a variance-covariance matrix derived from the patient-level data of the IND/GLY trials. ${ }^{7}$ The age and gender were set as from a normal distribution, while height, smoking status, and $\mathrm{FEV}_{1}$ status were then each derived using the variance-covariance matrix and a vector of standard normal variates. This process is known as the Cholesky decomposition, and more details can be found in the study by Briggs et al. ${ }^{21}$

The baseline lung function data $\left(\mathrm{FEV}_{1}\right)$ were set by patient's age, height, and gender which determined the expected normal value for a healthy individual with the same cohort characteristics. Then, the starting $\mathrm{FEV}_{1}$ was sampled using the variance-covariance matrix from the clinical trials and previous studies as provided in Table $1 .{ }^{17,22-26}$ Once a patient has been assigned a starting $\mathrm{FEV}_{1}$ value, there is a predetermined decline rate at every cycle depending upon the individual's smoking status. ${ }^{26}$ This value was also allowed to vary by treatment to take into account possible treatment effects.

Probabilities of death were determined by a hazard ratio taken from the OLIN study, ${ }^{27}$ and the general all-cause mortality was obtained from the abridged life table provided by the Department of Statistics, Ministry of the Interior. ${ }^{28}$

\section{Efficacy data}

Efficacy data (Table 2) were obtained from two Phase III head-to-head trials, ${ }^{12,13}$ and network analyses were used to compare the safety and efficacy of IND/GLY versus tiotropium and IND/GLY versus SFC. In the model, lung function improvement was captured as an increase in the actual $\mathrm{FEV}_{1}$ in liters from baseline, as determined using data from the ILLUMINATE ${ }^{12}$ and SPARK trials. ${ }^{13}$ We obtained the

Table 2 Efficacy data input

\begin{tabular}{|c|c|c|c|}
\hline Variables & Mean (L) & SE & Distribution \\
\hline \multicolumn{4}{|c|}{$\mathrm{FEV}_{\text {, improvement }}{ }^{12,13}$} \\
\hline IND/GLY & 0.26 & 0.026 & Normal \\
\hline Tiotropium & 0.13 & 0.016 & \\
\hline SFC & 0.16 & 0.013 & \\
\hline \multicolumn{4}{|c|}{ Exacerbation rate ratios ${ }^{12,13}$} \\
\hline IND/GLY & 0.61 & 0.10 & Lognormal \\
\hline Tiotropium & 0.91 & 0.10 & \\
\hline SFC & 0.75 & 0.10 & \\
\hline \multicolumn{4}{|c|}{ Withdrawal (assumption) } \\
\hline IND/GLY & 0.04 & & \\
\hline Tiotropium & 0.04 & & \\
\hline SFC & 0.04 & & \\
\hline
\end{tabular}

Abbreviations: $F E V_{1}$, forced expiratory volume in I second; GLY, glycopyrronium; IND, indacaterol; SFC, salmeterol/fluticasone combination. rate ratio of IND/GLY versus tiotropium directly from the SPARK study. ${ }^{13}$

However, since there is no direct comparison between IND/GLY and SFC, we therefore employed a network analysis from the placebo arms of the TORCH and ILLUMINATE trials. We calculated the rate ratio of IND/GLY versus SFC based on the raw rates of adverse events reported for each year in the ILLUMINATE study, as follows: IND/GLY (0.847 per year) and SFC (1.05 per year). The TORCH trial reported the following rates for either moderate or severe exacerbation: SFC (0.85 per year) and placebo (1.13 per year); thus, we derived a reported rate ratio of $0.75(95 \%$ CI: $0.69-0.81)$ for SFC versus a placebo. ${ }^{29}$ Based on the relationships reported in the TORCH trial, we calculated a hypothetical placebo exacerbation rate of 1.40 per year. This was in turn used to calculate the rate ratio of IND/GLY versus a placebo. In doing so, we calculated the exacerbation rate ratio of IND/GLY versus SFC. Thus, the exacerbation rate ratios, compared to placebo, were $0.61,0.91$, and 0.75 for IND/GLY, tiotropium, and SFC, respectively.

Each case of exacerbation was assigned a disease severity state according to the last pre-bronchodilator FEV , measure- $_{1}$ ment prior to the start date of the exacerbation. Exacerbations were further classified as either severe (requiring hospitalization) or non-severe (requiring contact with a health care provider for a change in therapy). The ratio of non-severe versus severe exacerbations by disease severity was obtained from the ad hoc analyses performed on the pooled placebo arm of clinical trials. ${ }^{20,30}$ The proportion of non-severe exacerbations in different COPD severities was 100\% in Global Initiative for Chronic Obstructive Lung Disease (GOLD) I, 94\% in GOLD II, 92\% in GOLD III, and $87.5 \%$ in GOLD IV.

\section{Cost data}

This study was conducted from the perspective of the third-party payer; therefore, we included only direct costs in our analysis. Costs associated with COPD maintenance, exacerbation, and drugs were obtained from NHI claims data (Table 2). To avoid double counting, we defined maintenance costs as non-exacerbation-related costs following the exclusion of COPD drug costs. The direct costs included other medications, outpatient care, examinations, and other costs. After adjusting for patients' age, gender, and follow-up year using mixed regression analysis, we used the adjusted costs associated with the severity of COPD in our analysis. We then recalculated the maintenance costs according to health status in each cycle. 
The exacerbation costs were subdivided into non-severe and severe, based on resource usage. Non-severe exacerbation was assumed to involve the costs associated with an outpatient visit and temporally needed to receive additional medication, including high-dose oral steroids $(>20 \mathrm{mg} /$ day) or antibiotics for at least 1 week. Severe exacerbation was assumed to involve the costs associated with admission into hospital.

Drug costs were translated into daily costs by dividing the cost of the drug by units per pack times the number of units used per day. Daily drug costs were then accumulated in each cycle depending on the therapy that COPD patient received. The cost of IND/GLY was increased or decreased by $25 \%$ when conducting sensitivity analysis.

\section{Utility data}

The calculation of utility was based on a regression model published by Rutten-van Molken et al. ${ }^{31}$ The covariates used to calculate utility included the patient's gender, postbronchodilator $\mathrm{FEV}_{1} \%$ predicted, the number of hospital admissions and emergency visits in the previous year, BMI, and comorbidities (Table 3 ).

Utility in each cycle was calculated using the following regression equation:

$$
\begin{aligned}
\text { Utility value }= & 0.688+\text { Gender } \times 0.057 \\
& +\mathrm{FEV}_{1} \text { percent predicted } \times 0.003 \\
& + \text { ER visits in the last year } \times 0.029 \\
& + \text { Hospital admission in the last year } \times-0.02 \\
& + \text { Number of concomitant disease } \times-0.01 \\
& + \text { BMI } \times-0.003
\end{aligned}
$$

Table 3 Cost inputs

\begin{tabular}{llll}
\hline Variables & Mean & SE & Distribution \\
\hline Maintenance cost (US\$) & & & \\
GOLD I & 210.65 & 14.16 & Gamma \\
GOLD II & 242.72 & 10.89 & Gamma \\
GOLD III & 213.09 & 28.74 & Gamma \\
GOLD IV & 341.83 & 28.60 & Gamma \\
Exacerbation cost (US\$) & & & \\
$\quad$ Non-severe exacerbation & 68.89 & 1.75 & Gamma \\
$\quad$ Severe exacerbation & $3,529.37$ & 120.18 & Gamma \\
Drug costs (daily cost, US\$) & & & \\
IND/GLY & 2.02 & & \\
Tiotropium & 1.67 & & \\
SFC & 1.50 & & \\
\hline
\end{tabular}

Abbreviations: GLY, glycopyrronium; GOLD, Global Initiative for Chronic Obstructive Lung Disease; IND, indacaterol; SFC, salmeterol/fluticasone combination.
Each utility value was assigned as per the disease state outlined in GOLD 2008, thereby disallowing distinctions in utility associated with smaller changes in the $\mathrm{FEV}_{1}$ level.

\section{Results \\ Base case}

Table 4 presents the results of base-case analysis under various comparator settings and time horizons. The lifetime life year (LY) and QALY in patients treated with IND/GLY were 11.48 and 6.95, respectively. Treatment with tiotropium and SFC resulted in QALY of 6.87 and 6.92, respectively. The incremental lifetime costs were as follows: IND/GLY versus tiotropium (US\$463) and IND/GLY versus SFC (US\$1,894). Since the incremental value of QALY were 0.08 and 0.13 ; thus, the ICER were US\$5,899 and US\$14,991 per QALY gained, respectively.

\section{Sensitivity analysis}

The results of one-way sensitivity analysis did not differ substantially from those of base-case analysis. All the results (Figure S1A and B) were less than three times the gross domestic product (GDP) per capita of US\$60,000/ QALY, which is the threshold suggested by the World Health Organization. ${ }^{32}$ When compared to tiotropium, the benefits of $\mathrm{FEV}_{1}$ from treatment, exacerbation rate ratio, and proportion of very severe COPD patients are the key drivers in the CE of IND/GLY. When compared to SFC, the exacerbation rate ratio and the proportion of very severe COPD patients largely determine the CE of IND/GLY.

Figure 2 shows the CE scatterplot of IND/GLY versus tiotropium and IND/GLY versus SFC. In a simulation that included 1,000 cohorts of 10,000 patients each, $99.2 \%$ of the points comparing IND/GLY versus tiotropium were located in the first and fourth quadrants of the CE plane. In comparison, $100 \%$ of the points comparing IND/GLY versus SFC were located in the first and fourth quadrants. The CEAC in Figure 3 shows that, setting the willingness-to-pay threshold per QALY gained at US\$20,000 (approximately 1 GDP per capita in 2016), there was a $97.8 \%$ probability that IND/GLY was cost-effective when compared to tiotropium and a $95.4 \%$ probability that IND/GLY was cost-effective when compared to SFC. When the WTP threshold was set at US $\$ 60,000$ (approximately three times the GDP per capita in 2016), there was a $98.7 \%$ probability that IND/GLY was cost-effective compared to tiotropium. Similarly, there was a $99.9 \%$ probability that IND/GLY was cost-effective compared to SFC. 
Table 4 Base-case CE results for IND/GLY versus tiotropium and IND/GLY versus SFC

\begin{tabular}{|c|c|c|c|c|c|c|c|c|c|c|}
\hline \multirow[t]{2}{*}{ Variables } & \multicolumn{5}{|c|}{ IND/GLY versus tiotropium } & \multicolumn{5}{|c|}{ IND/GLY versus SFC } \\
\hline & I year & 3 years & 5 years & 10 years & Lifetime & I year & 3 years & 5 years & 10 years & Lifetime \\
\hline Incremental cost (US\$) & 37 & 98 & 155 & 278 & 463 & 160 & 442 & 703 & 1,235 & I,894 \\
\hline Incremental LY & 0.00 & 0.00 & 0.00 & 0.04 & 0.12 & 0.00 & 0.01 & 0.01 & 0.05 & 0.19 \\
\hline Incremental QALY & 0.00 & 0.01 & 0.01 & 0.03 & 0.08 & 0.00 & 0.01 & 0.01 & 0.04 & 0.13 \\
\hline Exacerbations avoided & 0.04 & 0.14 & 0.24 & 0.43 & 0.66 & 0.04 & 0.14 & 0.24 & 0.43 & 0.66 \\
\hline ICER per LY gained & $|74,7| 4$ & 38,393 & 19,756 & 8,388 & 4,012 & 573,139 & || $3,02 \mid$ & 53,273 & 22,899 & 10,223 \\
\hline ICER per QALY gained & 48,224 & 27,266 & 19,070 & 10,626 & 5,899 & 142,046 & 77,973 & 52,212 & 29,015 & $|4,99|$ \\
\hline ICER per exacerbation avoided & 903.69 & 693.73 & 657.85 & 643.49 & 701.45 & $3,952.68$ & $3, \mid 14.21$ & $2,986.04$ & $2,854.85$ & $2,868.62$ \\
\hline
\end{tabular}

Abbreviations: CE, cost-effectiveness; GLY, glycopyrronium; ICER, incremental cost-effectiveness ratio; IND, indacaterol; LY, life year; QALY, quality-adjusted life year; SFC, salmeterol/fluticasone combination.
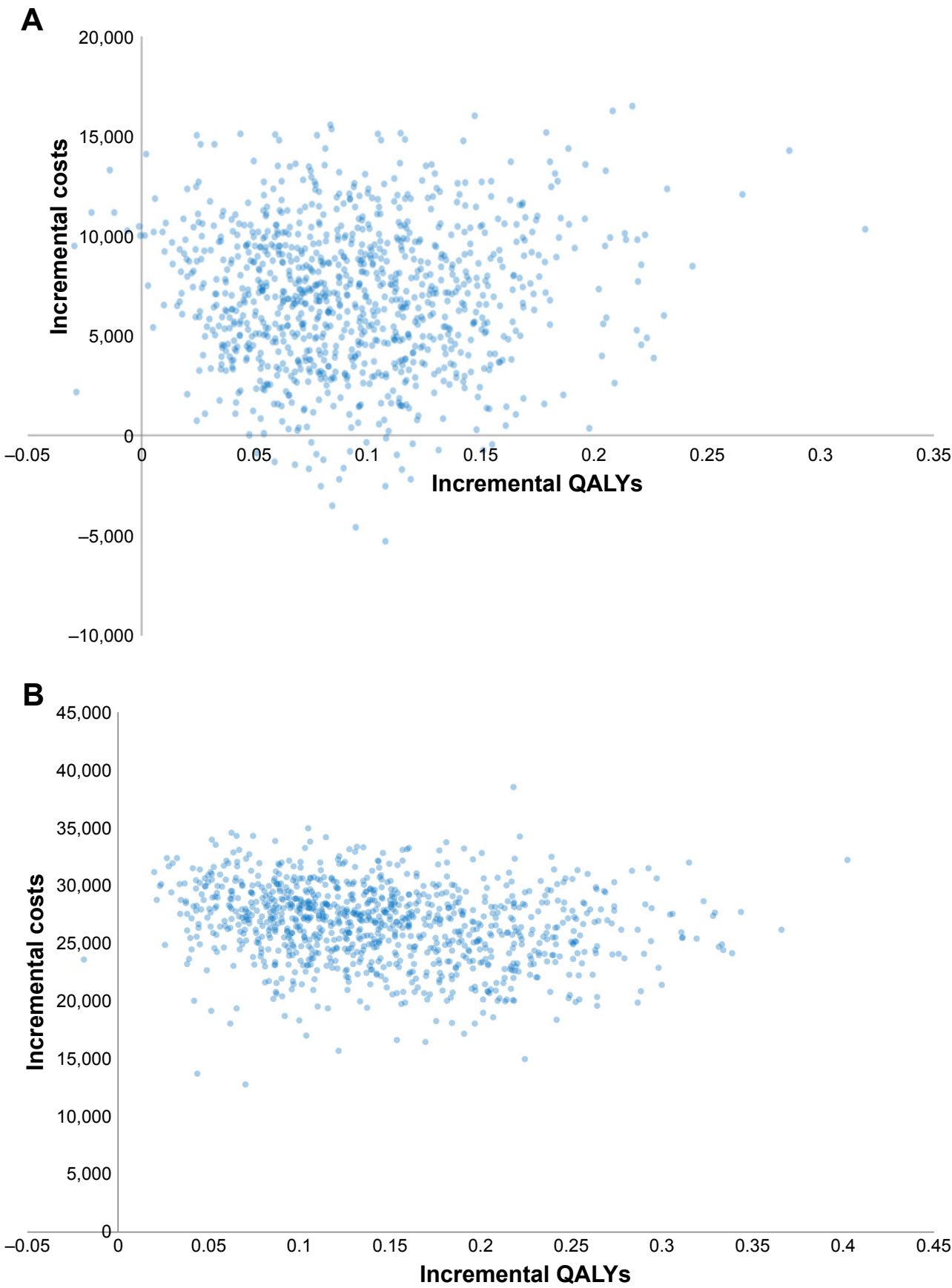

Figure 2 Incremental CE scatterplot showing cost per QALY gained through the administration of IND/GLY compared to (A) tiotropium and (B) SFC. Abbreviations: CE, cost-effectiveness; GLY, glycopyrronium; IND, indacaterol; QALY, quality-adjusted life year; SFC, salmeterol/fluticasone combination. 


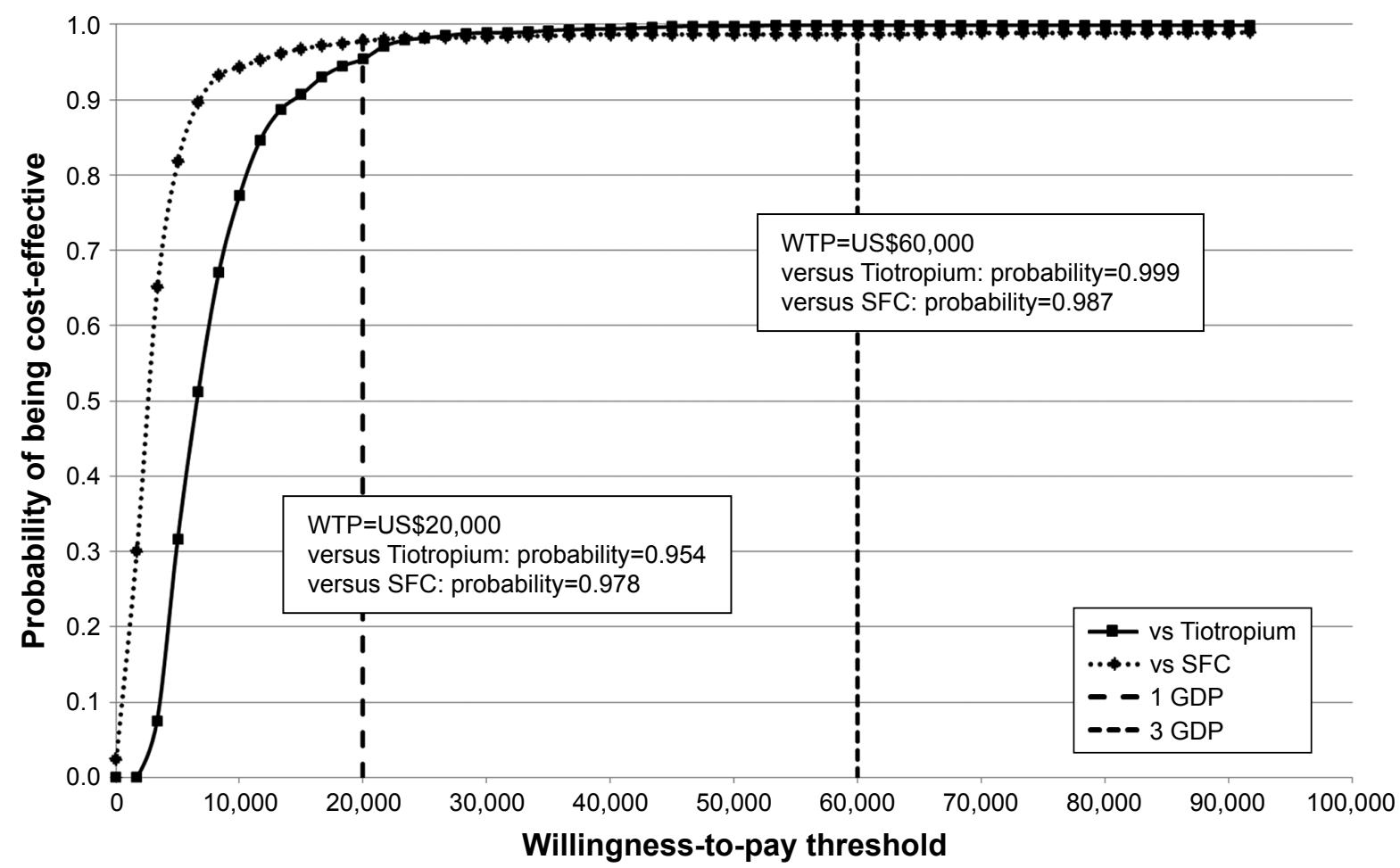

Figure 3 CEAC per QALY gained from IND/GLY.

Abbreviations: CEAC, cost-effectiveness acceptability curve; GDP, gross domestic product; GLY, glycopyrronium; IND, indacaterol; LABA, long-acting beta-2 agonist; LAMA, long-acting muscarinic antagonist; QALY, quality-adjusted life year; SFC, salmeterol/fluticasone combination; WTP, willingness-to-pay.

\section{Discussion}

There have been considerable advancements in our understanding of COPD, and several pharmacological treatments have been proposed. IND/GLY, as a dual bronchodilator, has potent pharmacological effects exceeding the efficacy of the single components in improving lung function and alleviating symptoms. In at least one previous study, IND/GLY was also shown to outperform ICS/LABA in preventing exacerbation. ${ }^{33}$

In this study, we evaluated the CE of IND/GLY versus tiotropium and IND/GLY versus SFC. We adopted the perspective of the third-party payer using costs derived from NHI claims data. IND/GLY, tiotropium, and SFC are all used in the maintenance treatment of COPD, and all three are fully reimbursed by the NHI. Our results show that implementing IND/GLY as the maintenance treatment for COPD patients would be considered as cost-effective than tiotropium or SFC. Among the improved outcomes, lives saved and reduced exacerbation events in the IND/GLY arm are particularly important ones. The probability that IND/ GLY would be a cost-effective alternative was estimated at $>95 \%$ for a WTP threshold exceeding US $\$ 20,000 /$ QALY. A price sensitivity analysis revealed that IND/GLY would remain cost-effective when compared to SFC, even in the event of a large price change. In addition, cohort model adopted by previous studies was limited to capture the key aspects of some prognoses, especially when the transition probabilities varied over time. In some situations, the transition probabilities increase or decrease with time (time dependency), which are hard to handle by cohort models. The individual sampling simulation (ISM) used in this study could simulate the time until the next event (eg, exacerbation) for a given simulated patient who can avoid modeling time and effect in unnecessary interim computation. ${ }^{34}$

Reza Maleki-Yazdi et a ${ }^{15}$ used a patient-level simulation model to compare the CE of IND/GLY with SFC in moderate-to-severe COPD patients in Canada, France, Italy, and Portugal. Their results demonstrated the CE of IND/ GLY over SFC for patients with moderate-to-severe COPD. Price et $\mathrm{al}^{14}$ compared the CE of IND/GLY against SFC as well as against a combination of IND plus GLY as the maintenance treatment for COPD patients in Swedish health care setting. Under the assumption of equal efficacy, they reported that IND/GLY provided a cost saving of EUR $\$ 85$ over IND plus GLY. Furthermore, IND/GLY was dominant when compared to SFC. However, the efficacy used in the study came only from one clinical trial ${ }^{12}$ which may underestimate or overestimate the efficacy of treatment. The other difference between Price et al's study and our study was that Price et al's study included moderate-to-severe COPD patient with the mean age at baseline of 63.3 years for simulating, whereas 
we simulated all COPD patients aged 40 years. Our results are consistent with previous studies that, when compared to SFC, IND/GLY was a CE alternative treatment for COPD patients. In addition, we compared the CE of IND/GLY versus tiotropium, which is another frequent prescription by physicians for moderate-to-severe COPD patients.

To the best of our knowledge, this study is the first to assess the CE of IND/GLY versus tiotropium and IND/GLY versus SFC in a broad COPD population (including mild, moderate, severe, and very severe COPD) using data from clinical trials. Nonetheless, the following limitations must be taken into account when interpreting our results. First, there was a lack of direct evidence of FEV $\mathrm{F}_{1}$ improvement and exacerbation reduction when comparing IND/GLY and SFC. The only available results were from comparisons of SFC versus placebo and IND/GLY versus placebo. Thus, our analysis is based on an indirect comparison using the baseline rates for a placebo in clinical trials. The other limitation is the fact that this study was based on the 2008 GOLD guidelines, in which the classification of severity was based on $\mathrm{FEV}_{1}$. We opted for this approach to be in parallel with the clinical trials used as a source of inputs. Future study could examine the impact of GOLD 2017 guidelines, in which the severity classification has been redefined according to exacerbation history, symptoms, and spirometry results, and then modify the CE model by including more disease characteristics in addition to $\mathrm{FEV}_{1}$.

The price of respiratory drugs was adjusted on April 1, 2017. The fact that the prices of SFC and tiotropium have been reduced could impact the results of our analysis. The magnitude of the price reduction was not known at the time of the study. Nonetheless, under the current parameters, even a $20 \%$ reduction in the price of SFC would still make IND/GLY a cost-effective alternative to SFC (ICER of US\$29,141/QALY gained). Our results also showed that IND/GLY would be cost-effective even if the drug price of IND/GLY increased or decreased by $25 \%$. The third-party payer's perspective was used in the study; furthermore, IND/ GLY, tiotropium, and SFC were both covered in the NHI in Taiwan. Thus, the generalizability of the results to other health care settings may be limited. This study was also limited by the fact that indirect costs were not included in our analysis, which would have provided a more complete picture of the true costs of COPD.

\section{Conclusion}

From the perspective of the health care payer in Taiwan, our results show that using IND/GLY as a maintenance treatment for COPD patients could be considered as a cost-effective alternative when compared to either tiotropium or SFC.

\section{Acknowledgment}

This study was sponsored by Novartis, Taiwan.

\section{Author contributions}

All the authors participated in the study design, data analysis, interpretation of the results, writing and reviewing of the manuscript, and decision to submit the manuscript for publication.

\section{Disclosure}

The authors report no conflicts of interest in this work.

\section{References}

1. Gardiner C, Gott M, Payne S, et al. Exploring the care needs of patients with advanced COPD: an overview of the literature. Respir Med. 2010; 104(2):159-165.

2. Murray CJ, Lopez AD. Alternative projections of mortality and disability by cause 1990-2020: Global Burden of Disease study. Lancet. 1997;349(9064):1498-1504.

3. Soler-Cataluna JJ, Martinez-Garcia MA, Roman Sanchez P, Salcedo E, Navarro M, Ochando R. Severe acute exacerbations and mortality in patients with chronic obstructive pulmonary disease. Thorax. 2005;60(11): 925-931.

4. Spencer S, Jones PW, Group GS. Time course of recovery of health status following an infective exacerbation of chronic bronchitis. Thorax. 2003;58(7):589-593.

5. Donaldson GC, Seemungal TA, Bhowmik A, Wedzicha JA. Relationship between exacerbation frequency and lung function decline in chronic obstructive pulmonary disease. Thorax. 2002;57(10):847-852.

6. Toy EL, Gallagher KF, Stanley EL, Swensen AR, Duh MS. The economic impact of exacerbations of chronic obstructive pulmonary disease and exacerbation definition: a review. COPD. 2010;7(3):214-228.

7. Calverley PM, Anderson JA, Celli B, et al. Salmeterol and fluticasone propionate and survival in chronic obstructive pulmonary disease. N Engl J Med. 2007;356(8):775-789.

8. Global Initiative for Chronic Obstructive Lung Disease (GOLD) [webpage on the Internet]. Global Strategy for the Diagnosis, Management and Prevention of COPD. 2017. Available from: http://goldcopd.org/ gold-2017-global-strategy-diagnosis-management-prevention-copd/. Accessed March 6, 2018.

9. White P, Thornton H, Pinnock H, Georgopoulou S, Booth HP. Overtreatment of COPD with inhaled corticosteroids - implications for safety and costs: cross-sectional observational study. PLoS One. 2013; 8(10): 75221.

10. Kew KM, Seniukovich A. Inhaled steroids and risk of pneumonia for chronic obstructive pulmonary disease. Cochrane Database Syst Rev. 2014;3:CD010115.

11. Bateman ED, Ferguson GT, Barnes N, et al. Dual bronchodilation with QVA149 versus single bronchodilator therapy: the SHINE study. Eur Respir J. 2013;42(6):1484-1494.

12. Vogelmeier CF, Bateman ED, Pallante J, et al. Efficacy and safety of once-daily QVA149 compared with twice-daily salmeterol-fluticasone in patients with chronic obstructive pulmonary disease (ILLUMINATE): a randomised, double-blind, parallel group study. Lancet Respir Med. 2013;1(1):51-60. 
13. Wedzicha JA, Decramer M, Ficker JH, et al. Analysis of chronic obstructive pulmonary disease exacerbations with the dual bronchodilator QVA149 compared with glycopyrronium and tiotropium (SPARK): a randomised, double-blind, parallel-group study. Lancet Respir Med. 2013;1(3):199-209.

14. Price D, Keininger D, Costa-Scharplatz M, et al. Cost-effectiveness of the LABA/LAMA dual bronchodilator indacaterol/glycopyrronium in a Swedish healthcare setting. Respir Med. 2014;108(12): 1786-1793.

15. Reza Maleki-Yazdi M, Molimard M, Keininger DL, et al. Cost effectiveness of the long-acting beta2-adrenergic agonist (LABA)/ long-acting muscarinic antagonist dual bronchodilator indacaterol/ glycopyrronium versus the LABA/inhaled corticosteroid combination salmeterol/fluticasone in patients with chronic obstructive pulmonary disease: analyses conducted for Canada, France, Italy, and Portugal. Appl Health Econ Health Policy. 2016;14(5):579-594.

16. Asukai Y, Baldwin M, Fonseca T, Gray A, Mungapen L, Price D. Improving clinical reality in chronic obstructive pulmonary disease economic modelling. Pharmacoeconomics. 2013;31(2):151-161.

17. Falaschetti E, Laiho J, Primatesta P, Purdon S. Prediction equations for normal and low lung function from the Health Survey for England. Eur Respir J. 2004;23(3):456-463.

18. Mahler DA, Wire P, Horstman D, et al. Effectiveness of fluticasone propionate and salmeterol combination delivered via the Diskus device in them treatment of chronic obstructive pulmonary disease. Am J Respir Crit Care Med. 2002;166(8):1084-1091.

19. Fenwick E, Marshall DA, Levy AR, Nichol G. Using and interpreting cost-effectiveness acceptability curves: an example using data from a trial of management strategies for atrial fibrillation. BMC Health Serv Res. 2006;6:52.

20. Dahl R, Jadayel D, Alagappan VKT, Chen U, Banerji D. Efficacy and safety of QVA149 compared to the concurrent administration of its monocomponents indacaterol and glycopyrronium: the BEACON study. Int J Chron Obstruct Pulmon Dis. 2013;8:501-508.

21. Briggs AH, Claxton K, Sculpher MJ. Decision Modelling for Health Economic Evaluation. Oxford: Oxford University Press; 2006.

22. Brandli O, Schindler C, Kunzli N, Keller R, Perruchoud AP. Lung function in healthy never smoking adults: reference values and lower limits of normal of a Swiss population. Thorax. 1996;51(3):277-283.

23. Ip MS, Ko FW, Lau AC, et al. Updated spirometric reference values for adult Chinese in Hong Kong and implications on clinical utilization. Chest. 2006;129(2):384-392.
24. Langhammer A, Johnsen R, Gulsvik A, Holmen TL, Bjermer L. Forced spirometry reference values for Norwegian adults: the Bronchial Obstruction in Nord-Trondelag study. Eur Respir J. 2001;18(5): 770-779.

25. Roca J, Burgos F, Sunyer J, et al. References values for forced spirometry. Group of the European Community Respiratory Health Survey. Eur Respir J. 1998;11(6):1354-1362.

26. Scanlon PD, Connett JE, Waller LA, et al. Smoking cessation and lung function in mild-to-moderate chronic obstructive pulmonary disease. The Lung Health study. Am J Respir Crit Care Med. 2000;161(2 pt 1): 381-390.

27. Lindberg A, Larsson LG, Muellerova H, Ronmark E, Lundback B. Up-to-date on mortality in COPD - report from the OLIN COPD study. BMC Pulm Med. 2012;12:1

28. Department of Statistics, Ministry of the Interior [homepage on the Internet]. Abridged Life Table 2013. 2013. Available from: http://sowf. moi.gov.tw/stat/year/y02-11.xls. Accessed April 29, 2014.

29. Vestbo J, Group TS. The TORCH (towards a revolution in COPD health) survival study protocol. Eur Respir J. 2004;24(2):206-210.

30. Dahl R, Jadayel D, Alagappan V, Chen H, Banerji D. Efficacy and safety of QVA149 compared to the concurrent administration of its monocomponents indacaterol and glycopyrronium: the BEACON study [Corrigendum]. International Journal of Chronic Obstructive Pulmonary Disease. 2014;9.

31. Rutten-van Molken MP, Oostenbrink JB, Tashkin DP, Burkhart D, Monz BU. Does quality of life of COPD patients as measured by the generic EuroQol five-dimension questionnaire differentiate between COPD severity stages? Chest. 2006;130(4):1117-1128.

32. World Health Organization. Cost-Effectiveness ThresholdsChoosing Interventions That Are Cost Effective (WHO-CHOICE). Available from: http://www.who.int/choice/cost-effectiveness/en/. Accessed March 13, 2018.

33. Zhong N, Wang C, Zhou X, et al. LANTERN: a randomized study of QVA149 versus salmeterol/fluticasone combination in patients with COPD. Int J Chron Obstruct Pulmon Dis. 2015;10:1015-1026.

34. Drummond M. Methods for the Economic Evaluation of Health Care Programmes. 4th ed. Oxford, UK; New York, NY: Oxford University Press; 2015. 


\section{Supplementary material}

A

\author{
$\mathrm{FEV}_{1}$ benefit (IND/GLY) \\ $\mathrm{FEV}_{1}$ benefit (Tiotropium) \\ Exacerbation rate ratio (IND/GLY) \\ Exacerbation rate ratio (Tiotropium) \\ Only moderate COPD severity
}

Only severe COPD severitty

Only very severe COPD severity

Cost of IND/GLY (+/- 25\%)

\section{ICER (US\$) per QALY gained}

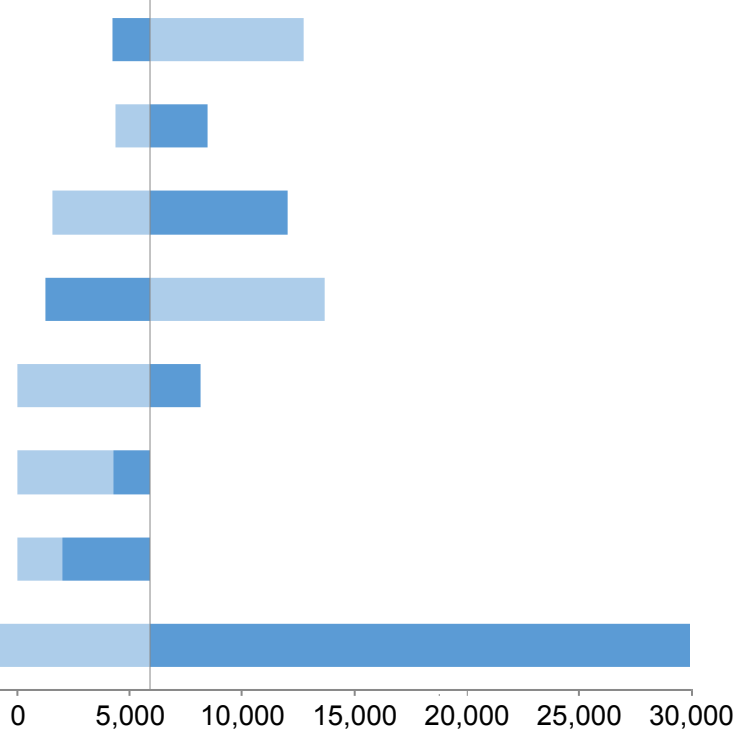

B

ICER (US\$) per QALY gained

$\mathrm{FEV}_{1}$ benefit (IND/GLY)

$\mathrm{FEV}_{1}$ benefit (SFC)

Exacerbation rate ratio (IND/GLY)

Exacerbation rate ratio (SFC)

Only moderate COPD severity

Only severe COPD severity

Only very severe COPD severity

Cost of SFC (-25\%)

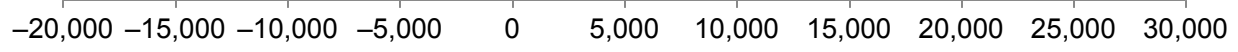

Figure SI One-way sensitivity analysis.

Note: (A) IND/GLY versus tiotropium and (B) IND/GLY versus SFC.

Abbreviations: $F E V_{1}$, forced expiratory volume in I second; GLY, glycopyrronium; ICER, incremental cost-effectiveness ratio; IND, indacaterol; QALY, quality-adjusted life year; SFC, salmeterol/fluticasone combination.

\section{Publish your work in this journal}

The International Journal of COPD is an international, peer-reviewed journal of therapeutics and pharmacology focusing on concise rapid reporting of clinical studies and reviews in COPD. Special focus is given to the pathophysiological processes underlying the disease, intervention programs, patient focused education, and self management protocols.

\section{Dovepress}

This journal is indexed on PubMed Central, MedLine and CAS. The manuscript management system is completely online and includes a very quick and fair peer-review system, which is all easy to use. Visit $\mathrm{http}: / /$ www.dovepress.com/testimonials.php to read real quotes from published authors. 